\title{
Defining the Role of the Dietitian in Dysphagia Assessment and Management
}

\section{Dietitians of Canada}

\section{EXECUTIVE SUMMARY}

This paper describes the dietitian's role in dysphagia assessment and management. Practice is based on the principles and ideals of evidence-informed, interprofessional, safe, competent and ethical client-centred care.

The dietitian's role is to provide services to meet the nutrition-care needs of individuals, and there is a direct relationship between swallowing function and nutritional status. Dysphagia assessment and management falls within the dietetic scope of practice in provincial legislation in all jurisdictions in Canada.

In dysphagia assessment and management, dietitians:

- provide client- and family-centred care; they respect and are responsive to client preferences, needs and culture, ensuring the client and family understand options for assessment and treatment, and provide informed consent;

- obtain and interpret information from mealtime/feeding observations, including estimation of daily nutrient intakes;

- identify signs and symptoms of dysphagia;

- assess the client's nutritional status and requirements for food, fluid and nutrition;

- identify the nutrition diagnoses such as swallowing difficulty;

- conduct or coordinate a clinical (bedside/tableside) swallowing assessment and/or instrumental swallowing assessment, where permitted by law;

- identify the risk of choking, aspiration and associated risk of pneumonia;

- consider the balance of risks and benefits of a swallowing assessment and treatment for individual clients;

- determine appropriate interventions and obtain client consent for diet, meal pattern, nutritional supplements and/or assistive devices as needed, food texture and liquid consistency modifications, positioning and the route of feeding;

- develop enteral and parenteral feeding regimes;

- provide client and/or family education and counselling;

- document and communicate the nutrition care process;

- monitor and evaluate a client's response to nutrition interventions, and modify the care plan as needed;

- act as a resource for clients and their care providers;

- work collaboratively and consult with other health care providers in the interprofessional team and circle of care; and

- facilitate ethical decision-making discussions, with the client's values guiding clinical decisions.

This statement reaffirms the professional practice obligation of the registered dietitian to meet client care needs by practicing to full scope, recognizing context and practice setting, while appreciating the value of interprofessional collaboration and effective team work.

(Can J Diet Pract Res. 2015;76:100)

(DOI: 10.3148/cjdpr-2015-013)

Published at dcjournal.ca on 19 May 2015.

A full statement can be accessed online at www.dietitians.ca/dysphagia

\section{APERÇU}

Ce document décrit le rôle des diététistes dans l'évaluation et la gestion de la dysphagie. La pratique s'appuie sur les principes et idéaux de soins interprofessionnels fondés sur des données probantes, sécuritaires, compétents, éthiques et axés sur le client.

Le rôle des diététistes est d'offrir des services visant à satisfaire les besoins des personnes en matière de soins nutritionnels. À cet égard, il existe une relation directe entre la fonction de déglutition et l'état nutritionnel. L'évaluation et la gestion de la dysphagie font partie du champ de pratique des diététistes dans la législation de toutes les provinces du Canada.

Dans le cadre de l'évaluation et de la gestion de la dysphagie, les diététistes

- Prodiguent des soins axés sur le client et sa famille; respectent les préférences, les besoins et la culture du client et y sont sensibles, s'assurant que ce dernier et sa famille comprennent les options d'évaluation et de traitement et qu'ils fournissent un consentement éclairé;

- Obtiennent et interprètent l'information issue d'observations effectuées lors des repas et de l'alimentation, y compris l'estimation des apports quotidiens en nutriments;

- Identifient les signes et les symptômes de la dysphagie;

- Évaluent l'état nutritionnel du client et ses besoins en aliments, en liquides et en nutriments;

- Déterminent les diagnostics nutritionnels tels que le trouble de déglutition;

- Réalisent ou coordonnent une évaluation clinique (au chevet) et/ou instrumentale de la déglutition, lorsque la loi le permet;

- Identifient les risques de suffocation et d'aspiration de corps étrangers de même que les risques associés de pneumonie;

- Tiennent compte de l'équilibre entre les risques et les avantages lié à l'évaluation de la déglutition et au traitement chez des clients donnés;

- Déterminent les interventions appropriées et obtiennent le consentement des clients concernant le régime alimentaire, le profil de repas, les suppléments nutritifs et/ou les dispositifs d'assistance, au besoin, les modifications à la texture des aliments et à la consistance des liquides, le positionnement et la voie d'alimentation;

- Mettent au point des régimes d'alimentation entérale et parentérale;

- Éduquent et conseillent les clients et/ou leur famille;

- Documentent et expliquent le processus de soins en nutrition;

- Surveillent et évaluent la réponse des clients aux interventions nutritionnelles, en plus de modifier le plan de soins au besoin;

- Agissent à titre de personnes-ressources auprès des clients et de leurs aidants naturels;

- Collaborent avec d'autres professionnels de la santé de l'équipe interprofessionnelle et du cercle de soins et leur demandent conseil; et

- Facilitent les discussions portant sur la prise de décisions éthiques, en s'appuyant sur les valeurs du client pour orienter les décisions cliniques.

Cet énoncé réaffirme l'obligation professionnelle des diététistes de répondre aux besoins des clients en matière de soins en utilisant l'ensemble de leur champ de pratique et en tenant compte du contexte et du milieu de pratique, tout en appréciant la valeur de la collaboration interprofessionnelle et du travail d'équipe efficace.

(Rev can prat rech diétét. 2015;76:100)

(DOI: 10.3148/cjdpr-2015-013)

Publié au dcjournal.ca le 19 mai 2015

L'énoncé complet peut être consulté au www.dietetistes.ca/dysphagie 